\title{
Reply to Andriy Vasylchenko's Review of Formal Ontology and Conceptual Realism
}

\author{
Nino B. Cocchiarella
}

Andriy Vasylchenko makes the interesting observation that our references are frequently emotionally charged. A comprehensive theory of reference, Vasylchenko suggests, should include an account of this phenomenon. We agree. Indeed, as we will see, the theory of reference in conceptual realism can be used to explain an important feature of our emotional states when we read a novel, or watch a play, a movie, or even when viewing a painting. This feature, which in aesthetics is called psychical distance, is connected in part with the difference between active and deactivated reference in conceptual realism. We will take up that issue at the end this reply.

There is, however, an important misunderstanding in Vasylchenko's review of how the notion of existential presupposition applies - or, as he claims, fails to apply - to fictional objects and more generally to the abstract intensional objects of conceptual realism. We will discuss this latter issue first, and then turn to the issue of our emotional states and psychical distance when reading fiction or watching a play or a film, and perhaps even when having an aesthetic experience in general.

\section{Existential Presupposition}

The central topic of conceptual realism, as Vasylchenko notes, is predication, which includes not only predication in language, but predication in thought and reality as well. The fundamental one of the three, I maintain, is predication in thought, which underlies predication in our speech and mental acts. Predication in reality, i.e., in states of affairs, is independent of predication in thought and language, but, on the conceptualist view, we are able to understand and represent it only by analogy with predication in thought.

The two fundamental parts of predication in thought are referential and predicable concepts, which are the cognitive capacities that underlie our rulefollowing abilities in the use of referential and predicate expressions, or what in natural language grammar are described as noun phrases and verb phrases, the two major parts of an indicative sentence. Noun phrases, of course, include not only proper names and definite descriptions, but quantifier phrases, such as 'Every painting', 'Some mountains', 'A unicorn', 'Most doctors', 'Few scholars', etc.

As cognitive capacities that are intersubjectively realizable, referential and predicable concepts can be exercised by different people at the same time as 
well as by the same person at different times. In other words, referential and predicable concepts are different types of "objective cognitive universals". In addition, referential and predicable concepts are complementary, unsaturated cognitive capacities, which when exercised together mutually saturate each other and result in a speech or mental act, i.e., a linguistic or mental event. The exercise of the predicable concept is what informs that act with a predicable nature; and the exercise of the referential concept is what informs the act with a referential nature.

The unsaturated nature of referential concepts corresponds to the way that quantifier phrases are unsaturated until they are applied to an open unsaturated predicate expression; and the unsaturated nature of a predicable concept corresponds similarly to an open, unsaturated predicate expression. Just as a quantifier phrase and a predicate expression mutually saturate each other and result in a sentence, so too referential and predicable concepts mutually saturate each other and result in a speech or mental act.

As referential expressions, proper names and definite descriptions initially are not singular terms on this account; rather, when used in a speech act, they are represented by a special type of quantifier phrase. ${ }^{1}$ It is appropriate to do so, moreover, because both a proper name and a definite description can be used either with or without existential presuppositions, and the way to represent either case is with a quantifier in what has come to be called "free logic" (i.e., logic free of existential presuppositions). ${ }^{2}$ Thus, for example, if the proper name 'Socrates' is used with existential presupposition in an assertion of the sentence 'Socrates is wise', then we can represent this by means of the existential quantifier as $(\exists x$ Socrates $) W i s e(x)$, which is equivalent to the more usual free-logic expression $(\exists x)[x=$ Socrates $\wedge$ Wise $(x)]$.

The two formulations are logically equivalent, but whereas the first can be used to represent the cognitive structure of the speech act in question, the latter can represent only the truth conditions of that act. On the other hand, an assertion of 'Pegasus is winged' in which the name 'Pegasus' is used without existential presupposition would be symbolized as $(\forall x$ Pegasus $)$ Winged $(x)$, which is equivalent to $(\forall x)[x=$ Pegasus $\rightarrow W$ inged $(x)]$. (A similar distinction is made in regard to the use of definite descriptions, but we will not go into that here.)

As these examples indicate, proper names, in the logical framework of conceptual realism, occur initially as parts of quantifier phrases; but then so do common names, i.e., common nouns, which include both count nouns such as 'unicorn' and 'elephant', and mass nouns such as 'furniture' and 'water'. Thus, an assertion of '(All) unicorns are herbivorous', would be symbolized as $(\forall x$ Unicorn $)$ Herbervivous $(x)$, which has the same truth conditions as

$$
(\forall x)[(\exists y U \text { nicorn })(x=y) \rightarrow \text { Herbivorous }(x)],
$$

\footnotetext{
${ }^{1}$ Proper names do become object terms in a secondary sense in that part of conceptual realism that contains the logic of classes as many. But then so too do common names.

${ }^{2}$ See Lambert 1991 for a collection of the more important papers on free logic. It should be noted, however, that unlike the analysis given in conceptual realism, proper names and definite descriptions are analyzed as singular terms in all of those papers.
} 
and which therefore, as with all universal statements in modern logic, carries no existential presupposition that there are any unicorns. ${ }^{3}$ I am not sure what Vasylchenko means in his discussion of this example when he says that the person who asserts this believes that there are unicorns. In any case, he is incorrect when he says that an assertion of '(All) elephants are herbivorous' would be symbolized as $(\exists x$ Elephant)Herbivorus $(x)$, which says instead that some elephant is herbivorus, as opposed to $(\forall x$ Elephant $) H e r b i v o r u s(x)$, which refers to all elephants. In both the assertion about all unicorns and the assertion about all elephants there is no existential commitment to either unicorns or elephants.

These examples also indicate how the conceptual realist theory of reference described in my book is based on two levels of analysis with two ways of representing logical forms, one that respects the cognitive structure of our speech and mental acts, and the other that is concerned with the truth conditions and deductive relations of those acts. The one level belongs to that part of semiotic known as pragmatics, and the other to the part we call semantics. Theories of reference in modern philosophical analysis do not generally make this distinction between levels of analysis, and, as a result, they are almost exclusively theories of singular reference, i.e., theories about the use of proper names and definite descriptions as singular terms.

\section{Being Versus Existence}

Existential presupposition is not necessarily about existence in conceptual realism, though it can be, and was originally taken to be so in extensional logic where there is no distinction between being and existence. In general, existential presupposition is about whether or not existential generalization can be applied to an object term, and that depends on what the values of the bound object variables of the existential quantifier are taken to be in a given formal ontology. In actualism, where being is the same as concrete existence, only concrete existents are values of the bound object variables. But, once tense and modal contexts are admitted, that is not an appropriate position for a conceptualist to take.

In conceptualism all of our speech and mental acts are understood to be inextricably temporal phenomena located in a specious present. Implicit in every such act are certain cognitive schemata regarding the past and the future as well as the present. In conceptualism, these cognitive schemata are represented by tense operators, and it is in terms of these operators that we can distinguish between what exists now from what did exist or what will exist, where, by existence, we mean concrete existence (and will take it to be so understood hereafter ${ }^{4}$ Thus, although Socrates did exist, he does not now exist, and,

\footnotetext{
${ }^{3}$ Strictly speaking, this formula symbolizes the different sentence 'Every unicorn is herbivorous'. But this is a difference that requires a discussion of my analysis of plurals that we will not go into here. The two sentences, even though they have the same truth conditions, represent different speech acts. For more on this, see chapter 11, "Plurals and the Logic of Classes as Many," of Cocchiarella 2008.

${ }^{4}$ For an account of tense operators, see chapter 2, "Time, Being and Existence," of Coc-
} 
strictly speaking, the statement that Socrates is wise should really be that Socrates was wise. ${ }^{5}$

Being is not the same as concrete existence in conceptual realism, because past and future objects, as well as objects that now exist, are taken as values of the object variables bound by $\exists$ and $\forall$. Existence in this framework is represented by the object variables bound by the different quantifiers $\exists^{e}$ and $\forall^{e}$. This difference between being and existence also involves a second-order distinction between predicable concepts in general and those that entail existence, i.e., those under which only (concrete) existents can fall - and which hereafter we will call e-concepts. ${ }^{6}$ Being wise, being an elephant, and even being a unicorn are e-concepts, for example, because an object must be in the concrete, physical world in order actually to fall under any of these concepts. ${ }^{7}$ Being an ancestor of everyone now existing, on the other hand, is of course not an e-concept; and there are many other similar concepts that do not entail existence (including the concept of being an object that does not now exist). With this distinction as basic, we can define existence as falling under an e-concept; that is,

$$
E !(x)={ }_{d f}\left(\exists^{e} F\right) F(x) .
$$

This analysis explains why existence, which of course is itself an e-concept, is so different from most predicable e-concepts. That is, unlike the the ordinary e-concepts of our commonsense framework, existence, being defined in terms of a totality to which it belongs, is an impredicative e-concept.

Do realia, i.e., the things that did, do, or will exist, comprise all of the beings there are in conceptual realism? Well, the part of the framework that deals with natural realism does recognize that we may want to assume natural possibilia as well, i.e., the concrete objects that could exist in nature at some time or other, but which in fact do not - such as the atoms of a transuranic element that might in fact never be produced by any supernova, but which, in principle, could be produced in nature's causal matrix - as well of course as the physical objects of a world alternative to ours in a many-worlds interpretation of quantum mechanics.

In addition to concrete possibilia, there are, as we will see, objects that are conceivably possible in fiction and imagination, but they will be abstract intensional objects and not possible concreta. With $\diamond$ understood as what is (causally) possible in nature, (possible) concrete objects in general are those that fall under the concept $[\lambda x \diamond E !(x)]$. Abstract objects, on the other hand, do not fall under the concept $[\lambda x \diamond E !(x)]$.

\footnotetext{
chiarella 2008 .

${ }^{5}$ In general we will ignore the use of tense operators, except when we are dealing with an explicitly temporal context.

${ }^{6}$ See chapter 4 of Cocchiarella 2008 for a discussion and development of this distinction between predicable concepts. Another full development of the logic of e-concepts and concepts in general can also be found in Cocchiarella 1969.

${ }^{7}$ Here we must distinguish between being a unicorn in a fictional, mythological story as opposed to being a unicorn simpliciter. It is the latter that entails existence. The former entails existence only within the story.
} 
In any case, because they range over past, present, and future objects, as well perhaps as objects that could be causally realized in nature, the quantifiers $\forall$ and $\exists$ are initially said to be possibilist quantifiers in conceptual realism. It is in terms of these quantifiers that quantifier phrases are taken as representing referential concepts; and it is in terms of the existential quantifier $\exists$ that we understand existential generalization, and in that sense existential presupposition as well. This logic, for reasons explained below, is free of existential presuppositions regarding object terms. Concrete existence, which is based on the quantifiers $\forall^{e}$ and $\exists^{e}$, is also free of existential presuppositions.

Now in terms of the difference between being and concrete existence, Vasylchenko's first premise of the paradox of nonexistence, namely:

$\left(\mathbf{P r}_{1}\right) \quad$ When (truthfully) denying the concrete existence of something (i.e., some being), we refer to what does not (now) exist.

is true in conceptual realism. The second premise, however, namely:

$\left(\mathbf{P r}_{2}\right) \quad$ One cannot refer to something (i.e., some being) that does not (now) exist.

is false in conceptual realism. In other words, with respect to concrete existence, conceptual realism is inflationist, and not deflationist.

Vasylchenko's alternative to $\left(\mathrm{Pr}_{2}\right)$, namely:

$\left(\mathbf{P r}_{2}^{*}\right) \quad$ One can refer to anything that is, i.e., has being.

is also true in conceptual realism; but then, it is also true in conceptual realism that one can refer only to what has being. In other words, conceptual realism is deflationist with respect to being.

This stronger deflationist thesis - contrary to what Vasylchenko seems to claim - is false of Meinong's theory of objects, where, unlike conceptual realism, there are even impossible objects, i.e., objects (Gegenstände) that are "outside of being" (ausserseiend). Indeed, in Meinong's ontology there are even "objects of which it is true that there are no such objects." 8

The round square, for example, is a concrete object in Meinong's ontology, and as such it is both round and square simpliciter. In conceptual realism, it is at best only an abstract, intensional object of fiction, as it is, e.g., in the story, "Romeo and Juliet in Flatland," which is discussed below in section 5 (and in my book, pp. 165f). As an abstract, intensional object, the round square is round and square only in fiction, and not simpliciter.

\section{Intensional objects}

Past, present, future and causally possible concreta are not the only objects "there are" in the ontology of conceptual realism, in other words. In addition, "there are" the "object"-fied contents of our concepts, i.e., the intensional objects we purport to represent when, e.g., instead of using 'wise' or 'triangular' as predicates, we nominalize and speak of wisdom, i.e., of being wise, or of triangularity, i.e., of being triangular, etc. By nominalization in language and in thought we purport to transform what is not an object, namely an unsaturated

\footnotetext{
${ }^{8}$ Meinong 1960, p. 82f.
} 
concept, into an object. What this transformation involves is a reification of the intensional content of that concept into the domain of objects - i.e., a reification of the truth conditions determined by that concept in different possible contexts of use. Formally, this transformation is unproblematic for most of the concepts of our commonsense framework, and that is because those concepts determine unproblematic truth conditions. But there are some concepts whose truth conditions lead to paradox when reified. The best known such concept is the one upon which Russell based his paradox, namely, the concept of being a concept that does not fall under itself.

In Russell's 1903 logical realist ontology, predicates stand for properties and relations instead of unsaturated concepts; and a nominalized predicate was understood by Russell to denote the same property or relation that the predicate stands for in its role as a predicate. In that case, the only way to avoid the paradox, is to formally acknowledge that there can be no such property as the Russell property that is not a property of itself. That means that in order to avoid a contradiction we must restrict the comprehension principle in which properties and relations are posited corresponding to complex predicates. Russell did this by constructing a theory of types in which predicates could not be meaningfully applied to themselves.

The system $\lambda H S T^{*}$, which is equivalent to the theory of simple types, retains Russell's original context of second-order logic with nominalized predicates, but avoids the paradox by restricting the comprehension principle by a metalinguistic typing device. The system $\lambda H S T^{*}$ described in my book is not a type theory, but a second-order logic in which predicates can be applied to themselves. But, like type theory, $\lambda H S T^{*}$ is not based on a logic that is free of existential presuppositions.

In conceptual realism there can be no doubt that we can form such a concept as the Russell concept of being a concept that does not fall under itself. In addition, because concepts are unsaturated cognitive structures, what we purport to represent when we nominalize a predicate is not the concept itself but a reification of the intensional content of that concept. A paradox can then be avoided in this framework by simply denying that we can consistently reify the intensional content of every concept.

What that means is that the first-order logic part of the framework of conceptual realism must be free of existential presuppositions with respect to the quantifier $\exists$. In other words, not every nominalized predicate can be taken as denoting an intensional object that can be the value of a variable bound by $\exists$. This means that some nominalized predicates are denotationless as abstract singular terms. In particular, even though the Russell predicate stands for a concept (as a value of the bound predicate variables), i.e., even though

$$
(\exists F)(F=[\lambda x(\exists G)(x=G \wedge \neg G(x))])
$$

is provable in conceptual realism on the basis of an unrestricted comprehension principle regarding the representation of predicable concepts, nevertheless it is also provable that the intensional content of such a concept cannot be reified 
(as a value of the object variables), i.e.,

$$
\neg(\exists x)(x=[\lambda x(\exists G)(x=G \wedge \neg G(x))])
$$

is also provable in conceptual realism, on pain otherwise of contradiction.

The question then is: which nominalized predicates can be taken without contradiction as denoting an abstract, intensional object? The answer is that we can obtain the system $H S T_{\lambda}^{*}$, which is essentially equivalent to $\lambda H S T^{*}$ (and hence to the theory of simple types), by a special axiom schema, $\left(\exists / H S C P_{\lambda}^{*}\right)$, that gives us all of the reified concepts of $\lambda H S T^{*}{ }^{9}$

Vasylchenko's claim that we can refer in conceptual realism to intensional objects, or what Vasylchenko calls "nonexistents," only without existential presuppositions is simply not true. The requirement that the first-order logic of the quantifiers $\forall$ and $\exists$ be free of existential presuppositions, and specifically that nominalized predicates can be used as abstract singular terms without existential presuppositions, is an essential part of this framework. ${ }^{10}$

There are many concepts in the framework of $H S T_{\lambda}^{*}$ that cannot be shown to be reifiable on the basis of the schema $\left(\exists / H S C P_{\lambda}^{*}\right)$. The Fregean concept $N$ of a natural number, which is defined as follows:

$$
N={ }_{d f}[\lambda x(\forall F)(F(0) \wedge(\forall y)(\forall z)[F(y) \wedge \operatorname{Successor}(y, z) \rightarrow F(z)] \rightarrow F(x))],
$$

cannot be shown to be reifiable, i.e., we cannot prove in $H S T_{\lambda}^{*}$ that $(\exists x)(N=x)$. But the slightly restricted alternative concept $N^{\prime}$, defined as:

$$
\begin{gathered}
N^{\prime}=_{d f}[\lambda x(\forall F)((\exists w)(F=w) \wedge F(0) \wedge(\forall y)(\forall z)[F(y) \wedge \operatorname{Successor}(y, z) \rightarrow \\
F(z)] \rightarrow F(x))],
\end{gathered}
$$

can be shown to be reifiable on the basis of the schema $\left(\exists / H S C P_{\lambda}^{*}\right)$, i.e., $(\exists x)\left(N^{\prime}=x\right)$ is provable in $H S T_{\lambda}^{*}$. Now although $(\forall x)\left[N(x) \rightarrow N^{\prime}(x)\right]$ is easily seen to be provable, the converse cannot be proved unless we assume that $N=N^{\prime}$. But this is provable, it turn out, if and only if $N$ is reifiable, i.e., iff $(\exists x)(N=x)$ is provable in $H S T_{\lambda}^{*}$. The concept $N$, it should be noted, allows for an unrestricted induction principle for the natural numbers, whereas the concept $N^{\prime}$ allows only for a restricted form of induction, i.e., a form that is restricted to reifiable concepts. At this point, it remains an open question whether or not $N$ is reifiable in $H S T_{\lambda}^{*}$, i.e., whether or not $(\exists x)(N=x)$ is consistent in $H S T_{\lambda}^{*}$.

The reason Vasylchenko calls the intensional objects of conceptual realism "nonexistents", incidentally, is that as abstract objects it is impossible for them to fall under the concept of concrete existence; that is,

$$
(\forall F) \neg \diamond E !(F)
$$

\footnotetext{
${ }^{9}$ For a detailed description of $\left(\exists / H S C P_{\lambda}^{*}\right)$, see, e.g., page 299 of Cocchiarella 1986; or pages $106 \mathrm{f}$ and 179 of Cocchiarella 1987.

${ }^{10}$ Because abstract objects are now values of the variables bound by $\forall$ and $\exists$, it is inappropriate to call $\forall$ and $\exists$ possibilist quantifiers. But it is difficult to think of an alternative. Certainly "being quantifiers" is awkward.
} 
is a basic ontological thesis of conceptual realism. ${ }^{11}$

Vasylchenko also describes the logical framework of conceptual realism as a type theory. But as already noted, both of the systems $\lambda H S T^{*}$ and $H S T_{\lambda}^{*}$ are second-order predicate logics with nominalized predicates as abstract singular terms. They are not a form of type theory in which paradox is avoided by restricting predicates to different levels or types in the object language itself. ${ }^{12}$

\section{Deactivated Referential Concepts}

Nominalization is the linguistic counterpart of a pattern of reflexive abstraction in which we attempt to represent what is not an object-namely, an unsaturated cognitive capacity underlying our use of a predicate expression - as if it were an object. It is in this way, according to conceptual realism, that we are able to conceptually "grasp" and have knowledge of abstract objects as the intensional contents of our predicable concepts.

It is not just the intensional content of (most) predicable concepts that can be reified in conceptual realism, however. So too can the intensional content of referential concepts. And just as the nominalization of a predicate expression (or the nominalization of a predicable concept in thought) amounts to a deactivation of the primary role of that predicate (or concept), i.e., its role as a predicate, so too can referential expressions be deactivated. The quantifier phrase 'a unicorn' in an assertion of the sentence 'Sophia seeks a unicorn', for example, is not being used actively to refer to a unicorn, as it would purport to be, e.g., in an assertion of 'A unicorn is in the garden.' And the same is true in conceptual realism of all referential expressions that occur as parts of (complex) predicate expressions or of nominalized sentences, as in 'desires to be a president who is admired by everyone', and 'is loved by every man who loves a woman who does not love herself', as well as those that occur within the scope of a propositional attitude, such as 'believes that a unicorn is in the garden'.

The deactivation of a referential concept is based on a double reflexive abstraction in which a referential concept is first transformed into a predicable concept, which then is transformed in turn into an abstract object. The idea is that just as we are able to conceptually "grasp" the intensional content of a predicable concept by transforming that concept into an abstract object, so too we are able to conceptually grasp the intensional content of a referential concept by first transforming that referential concept into a predicable concept, and then transforming that predicable concept into an abstract object.

Thus, e.g., we can transform the referential concept represented by the quantifier phrase 'a unicorn' into the predicable concept of being a concept under which a unicorn falls as follow:

$$
[\lambda x(\exists F)(x=F \wedge(\exists y \text { Unicorn }) F(y))],
$$

which we can abbreviate by simply placing brackets around the quantifier phrase,

\footnotetext{
${ }^{11}$ See, e.g., page 123 of Cocchiarella 2008.

${ }^{12}$ The typing that is used in the schema $\left(\exists / H S C P_{\lambda}^{*}\right)$ is a metalanguage device analogous to that of Quine's set theories $N F$ and $M L$.
} 
as follows: $[\exists y U n i c o r n]$. That is,

$$
[\exists y \text { Unicorn }]=_{d f}[\lambda x(\exists F)(x=F \wedge(\exists y U n i c o r n) F(y))] .
$$

The sentence 'Sophia seeks a unicorn' can then symbolized as:

$$
(\exists x \operatorname{Sophia}) \operatorname{Seeks}(x,[\exists y \text { Unicorn }]),
$$

where, of course, it is understood that 'Sophia' is being used with existential presupposition. ${ }^{13}$

All quantifier phrases occurring as parts of (complex) predicate expressions are understood to be deactivated in this way; i.e., they are not being actively used to refer whenever they occur as part of a complex predicate expression or a nominalized sentence. Of course, unlike the relational predicate 'seeks', some relational predicates may be extensional in their second-argument position, as is the case, e.g., with 'finds'. In such a case, we need to add a meaning postulate in order to draw out some of the logical consequences of the predicate. Thus, because the meaning postulate for 'finds' would be:

$$
[\lambda x \operatorname{Finds}(x,[\exists y U \text { icorn }])]=[\lambda x(\exists y U \text { nicorn }) \operatorname{Finds}(x, y)],
$$

we can, e.g., infer that John believes that there is a unicorn in the garden if he believes that Sophia finds a unicorn in the garden. Of course, John might well not believe that there is a unicorn in the garden if he only believes that Sophia is seeking a unicorn in the garden.

\section{The Ontology of Fictional Objects}

Vasylchenko is correct in noting that referential expressions in fictional contexts are understood to be deactivated in conceptual realism. All of the people and events we read about in a fictional story are assumed to exist only within the context of the story; and for that reason all references in the story to people and events are implicitly understood to be deactivated. Fictional objects, on this account, are intensional objects: namely, the reified intensional contents of the referential expressions used to refer to those objects within the fiction.

Actually, it is not just in fiction that referential expressions are deactivated. This is true even when reading a newspaper story or listening to the news on the radio or television. The difference is that when we believe a story that we are reading, or being told, we "reactivate" the referential expressions by understanding the statements made in the story to be true. This is reflected by the formula

$$
\operatorname{True}([\varphi]) \leftrightarrow \varphi,
$$

which of course is a valid thesis. Here, the bracketed sentence $\varphi$ is nominalized, and hence all referring expressions within it are deactivated, whereas they are in a "reactivated" position in the occurrence of $\varphi$ on the right-hand side of the biconditional.

\footnotetext{
${ }^{13}$ We might also note that 'seeks' like most, if not all, verbs entails (concrete) existence in its first argument position (but not also in its second).
} 
Vasylchenko is correct in noting that each story can be viewed as a set of propositions, though, strictly speaking, in conceptual realism a story is not a set of propositions but a class as many of propositions. ${ }^{14}$ Vasylchenko is incorrect, however, in saying that we can associate a possible world with the set of propositions that make up a story. That is because possible worlds, but not also stories, are made up of maximally consistent sets of propositions. Stories, even when they are consistent, do not need to be maximally consistent, and some can be inconsistent. Indeed, as already noted, some stories can even have impossible objects among their characters, as, e.g., the round square in the story of Romeo and Juliet in Flatland already mentioned. In that story, Juliet is a circle (she has curves), and Romeo is a square (he has angles). Their families are always feuding, but Romeo and Juliet meet and have an affair. Juliet becomes pregnant and in time gives birth to a round square. The round square is viewed as a monster by both families and is destroyed, its birth kept a secret from the world. Romeo and Juliet are also killed, but they are passed off by their families as ill-starred lovers who committed suicide.

Of course the fact that there is a round square in the story does not mean that "there is" a round square simpliciter. Rather, it only mean that the intension of the concept of a round square can be reified within a story, just the way that the concept of a unicorn was reified by James Thurber in his well-known fable, The Unicorn in the Garden. ${ }^{15}$ This example illustrates how different our conceptual realist account of fictional and so-called impossible objects is from Meinong's in whose theory of objects "there is" a round square simpliciter.

Finally, Vasylchenko cites Terry Parsons's and E. Zalta's reconstructions of Meinong's theory of objects. It is noteworthy that in 1982 I showed how Parsons's reconstruction can be reinterpreted, and in that sense reduced, to Russell's 1903 ontology as reconstructed in $\square \lambda H S T^{*}$, a system equivalent to $H S T_{\lambda \square}^{*}$, the logical framework for conceptual realism. ${ }^{16}$ In fact, that reinterpretation also provides a useful reductive account of Meinong's unexplained distinction between nuclear and extranuclear properties in terms of the distinction between e-concepts and concepts in general. In 1990, Greg Landini showed how E. Zalta's reconstruction of Meinong's theory can also be reduced to Russell's 1903 ontology as reconstructed in terms of $\square \lambda H S T^{*}$, and therefore implicitly to $H S T_{\lambda \square}^{*}$ as well. ${ }^{17}$ In other words, these two papers show how Meinong's theory of objects, as reconstructed by Parsons and Zalta, can be reduced to, and explained in terms of, the framework of conceptual realism without any of the odd consequences of Meinong's ontology.

\section{Deactivation as Psychical Distance}

Vasylchenko notes that our use of referential concepts, whether in stories, day dreams, our imagination, or even in our everyday life, are often emotionally

\footnotetext{
${ }^{14}$ For a logic of classes as many and it uses in the analysis of plurals, see chapter 11 of Cocchiarella 2008. For an analysis of mass nouns in the same logic, see Cocchiarella 2009.

${ }^{15}$ The fable was first published in 1939 and later reprinted in a number of anthologies.

${ }^{16}$ See Cocchiarella 1982.

${ }^{17}$ Landini 1990.
} 
charged. Such an emotional charge is often an important part of an aesthetic experience in which we switch from a state of deactivated reference regarding a representation to a state of activated reference in which what is represented has taken on a reality of its own.

Sometimes, for example, we become so emotionally caught up in a story we are reading, or a play or film we are watching, or even a painting that we are viewing, that we lose what in aesthetics has come to be called psychical distance, a suspension of disbelief in which we enter the world being represented in the story, play, film, etc. ${ }^{18}$ When we lose psychical distance we are not only engaged more emotionally in what we are reading, watching, etc., but we also switch from a mental state of deactivated reference in which objects in the work are merely representations, i.e., intensional contents, to an emotionally charged state of active reference in which the objects are experienced as real, even if only momentarily.

We may experience fear, e.g., when watching a horror film, and in those brief moments when the fear has gripped us, our mental state may switch, and even flutter back and forth, between a state of deactivated reference and a state of active reference in which the frightening creature is taken as real. We might switch, in other words, between an adrenaline pumping state of active reference and a pressure-releasing state of deactivated reference in which we reassure ourselves that it is only a film we are watching and not something real. The different frequencies of the on-off switch, i.e., of the flutter between active and deactivated reference, explains the different psychical distances we might experience; in other words, the varying frequencies explain why it may seem as though there is a continuum between over-distancing and under-distancing between oneself and the events being portrayed in the film.

So too, the characters in a novel we may be reading, or a television soap opera we are watching, sometimes become so real to us that we begin to view them and their relations to one another as actual people in real situations; and we might even begin to admire, or despise, those characters. ${ }^{19}$ When that happens we are often, even if only briefly, in a state of active reference. As our psychical distance changes while undergoing an aesthetic experience, in other words, so too our mental state may change from one in which our references are fully activated to one in which they are fully deactivated, and the changes may flutter back and forth, giving us an emotional charge that we may enjoy or sometimes strongly dislike.

There is much more to an aesthetic experience when undergoing a change in psychical distance than just activated and deactivated reference, to be sure; but in regard to our present semantic-ontological interest in the nature of reference to fictional characters, the connection between the phenomenon of psychical distance and the switch between active and deactivated reference is important and deserves more study and discussion than we can give it here.

\footnotetext{
${ }^{18}$ This notion was first introduced by Edward Bullough in Bullough 1912.

${ }^{19}$ Actors playing medical doctors in soap operas often receive mail from fans asking for medical advice. The fans persist in viewing the characters portrayed as real even after turning off their televisions.
} 


\section{References}

[1] Bullough, Edward, 1912, "'Psychical Distance' as a Factor in Art and as an Aesthetic Principle," British Journal of Psychology, vol. 5 (1912): 87-117. For selections from this article, see http://www.csulb.edu/ jvancamp/361_r9.html.

[2] Cocchiarella, Nino B., 1969, "A Completeness Theorem in Second-Order Modal Logic," Theoria, vol. 2 (1969): 81-103.

[3] Cocchiarella, Nino B., 1982, "Meinong Reconstructed Versus Russell Reconstructed," Journal of Philosophical Logic, vol. 11 (1982): 183-214. (Reprinted in Cocchiarella 1987.)

[4] Cocchiarella, Nino B., 1986, Logical Investigations of Predication Theory and the Problem of Universals, Bibliopolis Press, Naples.

[5] Cocchiarella, Nino B., 1987, Logical Studies in Early Analytic Philosophy, Ohio State University Press, Columbus.

[6] Cocchiarella, Nino B., 2008, Formal Ontology and Conceptual Realism, Springer, Synthese Library Series, vol. 339, Dordrecht.

[7] Cocchiarella, Nino B., 2009, "Mass Nouns in a Logic of Classes as Many," forthcoming in Journal of Philosophical Logic.

[8] Lambert, Karel 1991, Philosophical Applications of Free Logic, Oxford University Press, Oxford.

[9] Landini, Gregory, 1990, "How to Russell Another Meinongian: A Russellian Theory of Fictional Objects Versus Zalta's Theory of Objects," Grazer Philosophische Studien, vol. 37 (1990): 93-122.

[10] Meinong, Alexis, 1960, "The Theory of Objects," translated from1904 original by R. Chisholm, in Realism and the Background of Phenomenology, ed. by R. Chisholm, The Free Press, Glencoe, ILL.

[11] Thurber, James, 1939, "The Unicorn in the Garden," The New Yorker, October 31, 1939. 